\title{
CARCINOMA EPIDERMOIDE INTRAORAL EM PALMAS, TOCANTINS: UMA ANÁLISE DE INCIDÊNCIA
}

\author{
INTRAORAL SQUAMOUS CELL CARCINOMA IN PALMAS, \\ TOCANTINS: AN INCIDENCE ANALYSIS
}

Paula Duran Marquez de Sousa ${ }^{1}$, Hugo Henrique Pereira Vaz Filho ${ }^{1}$, Ronyere Olegário Araújo ${ }^{2}$, Eduardo Zambaldi da $\mathrm{Cruz}^{3}$, Cristiano Abdalla Rosa ${ }^{4}$, Ana Cláudia Garcia Rosa 5 .

\begin{abstract}
RESUMO
Introdução: o carcinoma epidermoide oral (CE) é um neoplasma com diferenciação escamosa originado no epitélio das mucosas da cavidade bucal, além de ser a neoplasia maligna de cabeça e pescoço mais comum, excluindo-se os carcinomas de pele nãomelanomas. Os Registros de Câncer de Base Populacional (RCBP) são sistemas de informações relacionados ao Instituto Nacional do Câncer (INCA), específicos para a oncologia, que coletam, classificam e analisam os casos novos de câncer diagnosticados em uma área geográfica específica. Objetivo: realizar uma análise do RCBP da cidade de Palmas, Tocantins, Brasil, em um período de seis anos, comparado com o número de casos diagnosticados em um único serviço de atendimento municipal (Centro de Especialidades Odontológicas- CEO), no mesmo intervalo de tempo. Método: foi realizada a análise quantitativa comparando os dados do RCBP referentes aos anos 2008-2013 com os casos diagnosticados no CEO nos anos 2014-2019. Resultado: não houve diferença entre a quantidade de casos registrados no RCBP e no CEO nos períodos avaliados. Em relação à raça, no RCBP houve predomínio da raça parda, enquanto no CEO houve predomínio da raça branca; em relação à localização das lesões, no RCBP houve predomínio de CE em base de língua, enquanto no CEO houve predomínio de lesões em outras localizações. Conclusões: existem duas hipóteses: (1) os dados referentes à incidência de CE na população de Palmas podem estar subestimados, já que, em um mesmo intervalo de tempo, um único serviço da cidade (CEO) registrou o mesmo número de casos notificados no último RCBP; ou (2) houve um aumento real incidência de CE na população de Palmas, o que poderá ser evidenciado com a próxima divulgação de dados de registro do RCBP no período correspondente aos registros do CEO (2014-2019). Desta forma, ressalta-se a importância da notificação da doença pelos profissionais aos setores responsáveis.

Palavras-chave: carcinoma de células escamosas; boca; notificação de doenças; epidemiologia; incidência
\end{abstract}

\begin{abstract}
Introduction: oral squamous cell carcinoma (EC) is a neoplasm with squamous differentiation originating in the mucosal epithelium of the oral cavity, in addition to being a more common malignant neoplasm of the head and neck, excluding non-melanoma skin carcinomas. Population-based Cancer Registries (PBCR) are information systems related to the National Cancer Institute $(\mathrm{NCl})$, specific to oncology, which collect, classify and analyze new cases of cancer diagnosed in residents of a specific geographic area. Objective: to carry out an analysis of the RCBP of the city of Palmas, Tocantins, Brazil, over a period of six years, compared with the number of cases diagnosed in a single municipal care service (Center of Dental Specialties - CEO), in the same interval of time. Method: a quantitative analysis was performed comparing the RCBP data for the years 2008-2013 with the cases diagnosed at the CEO in the years 2014-2019. Result: there was no difference between the number of cases registered with the RCBP and the CEO in the evaluated periods. Regarding race, in the RCBP there was a predominance of brown race, while in the CEO there was a predominance of white race; in relation to the location of the lesions, in the RCBP there was a predominance on tongue basis, while in the CEO there was a predominance of lesions in other locations. Conclusions: there are two hypotheses: (1) the data referring to the incidence of EC in the population of Palmas may be underestimated, since, in the same time interval, a single city service (CEO) registered the same number of cases reported in the last RCBP; or (2) there was a real increase in the incidence of EC in the population of Palmas, which can be evidenced with the next release of RCBP registration data in the period corresponding to the CEO records (2014-2019). Thus, the importance of reporting the disease by professionals to the responsible sectors is emphasized.
\end{abstract}

Keywords: carcinoma; squamous cell carcinoma; mouth; disease; notification; epidemiology. incidence
Conflito de interesses: os autores declararam que não existem conflitos de interesses.
Direitos Autorais: (c) 2021 Sousa et al. Este é um artigo de acesso aberto que permite restrições em qualquer meio, desde que o autor original e a fonte sejam creditados. \begin{abstract}
RO, Cruz EZ, Santos FP, Rosa ACG (2021) Manisfestação primária de sífilis em
\end{abstract} Editor: Rosa A. C. G. Medicina, Universidade Federal do Tocantins, Brasil.

Publicado: 25 de julho de 2021. 
Geral de Palmas (HGP; atenção terciária em saúde bucal, no âmbito Estadual).

O carcinoma epidermoide oral (CE) é um carcinoma com diferenciação escamosa que tem origem no epitélio das mucosas da cavidade oral ${ }^{1}$, sendo a neoplasia maligna de cabeça e pescoço mais comum, excluindo-se os carcinomas de pele não-melanomas ${ }^{2}$.

Apesar da proximidade anatômica, sabe-se que CEs de cavidade oral e orofaringe são distintos e únicos, diferindo quanto à etiopatogenia [na orofaringe, os CE por vezes se relacionam à presença do papilomavirus humano (HPV)], tratamento e prognóstico ${ }^{2 ; 3}$. A cavidade oral compreende as mucosa labial, jugal, soalho de boca, rebordo alveolar e gengiva, dois terços anteriores da língua (anteriores às papilas circunvaladas), palato duro e trígono retromolar. A orofaringe, por sua vez, consiste no palato mole, terço posterior da língua (base da língua), tonsilas palatinas, pregas do palatoglosso, valécula e parede posterior da faringe ${ }^{2}$.

Em 2017, a OMS reconheceu as diferenças entre CE de cavidade oral e orofaringe e, a partir de 2017, passou a discutir esses tumores como entidades separadas, distinguindo também os carcinomas de orofaringe HPV- positivos dos HPV-negativos, já que esses últimos possuem pior prognóstico ${ }^{1}$. Os carcinomas do vermelhão do lábio, embora sejam comumente discutidos junto aos carcinomas intraorais ${ }^{1}$ também têm etiopatogenia distinta, pois não se relacionam diretamente ao tabagismo ou etilismo, mas à exposição solar crônica, sendo geralmente precedidos pela presença de queilite actínica (queilite solar). Além disso, possuem prognóstico muito mais favorável que os carcinomas intraorais e de orofaringe ${ }^{2}$.

Segundo as informações do Globocan, no mundo, em 2018 foram estimados 246 mil casos novos de cânceres de língua e cavidade oral (CID C00-C06) em homens e 108 mil em mulheres, sendo mais comum em homens acima dos 40 anos do que em mulheres. O risco estimado para essa neoplasia foi de 6,4/100 mil em homens e 2,9/100 mil em mulheres. Essas neoplasias são mais frequentes nos países do Sul da Ásia ${ }^{4}$.

No Brasil, em 2017, ocorreram 4.923 óbitos por câncer da cavidade oral em homens e 1.372 óbitos em mulheres, esses valores correspondem ao risco de 4,88/100 mil homens e $1,33 / 100$ mil mulheres ${ }^{5}$. Os fatores de risco mais conhecidos incluem o tabagismo e o consumo excessivo de álcool, sendo que o risco é 30 vezes maior para os indivíduos que fumam e bebem do que para aquelas pessoas que não o fazem ${ }^{5 ; 6}$.

Entre outros fatores de risco, encontra-se a exposição ao sol sem proteção (importante risco para o câncer de lábio), o excesso de gordura corporal, a infecção pelo HPV (relacionada ao câncer de orofaringe) e fatores relacionados à exposição ocupacional ${ }^{5}$.

Em Palmas, Tocantins, os serviços públicos de diagnóstico do carcinoma epidermoide oral compreendem: o Centro de Especialidades Odontológicas de Palmas (CEO; atenção secundária em saúde bucal, no âmbito Municipal) e o Hospital
O carcinoma epidermoide oral é uma doença de notificação compulsória (Lei 13.685, de 25 de junho de 2018) e deve ser realizada pelos profissionais de saúde aos setores responsáveis, o que é consolidado por meio dos Registros de Câncer de Base Populacional (RCBP) ${ }^{7}$. Os RCBPs são sistemas de informações relacionados ao Instituto Nacional do Câncer (INCA), específicos para a oncologia, que coletam, classificam e analisam os casos novos de câncer diagnosticados em moradores de uma área geográfica específica, permitindo conhecer a incidência de câncer daquela área. As notificações registradas no RCBP permitem uma ampla coleta de dados epidemiológicos pelo Ministério da Saúde e constitui numa das principais fontes de informação para a definição das políticas públicas da Atenção Oncológica, assim como para o desenvolvimento de estudos e pesquisas na área ${ }^{7}$. Embora os RCBPs sejam meios confiáveis de registro sobre a ocorrência de casos por região, acredita-se que os dados referentes à incidência de carcinoma epidermoide oral na cidade de Palmas, Tocantins, possam estar subestimados.

Desta forma, o presente trabalho tem por objetivo comparar os dados mais recentes relativos a incidência de $C E$, atualmente disponíveis no RCBP, para a cidade de Palmas, Tocantins, (20082013) com uma série de casos diagnosticados em um dos serviços de referência (CEO), em um mesmo intervalo de tempo (2014-2019), verificando a incidência do CE nesse intervalo e analisando as variáveis sexo, raça e localização das lesões orais (CID C00-C06).

\section{METODOLOGIA}

Este trabalho foi aprovado pelo comitê de Ética em Pesquisa da Fundação de Saúde Pública de Palmas, sob número CAAE 31352220.0.0000.9187.

Para a coleta de dados, foram consultados os prontuários eletrônicos dos pacientes que tiveram o diagnóstico de carcinoma epidermoide oral (CE), do Centro de Especialidades Odontológicas de Palmas (CEO). Foram incluídos na pesquisa todos os casos de CE diagnosticados na cavidade oral (CID COOC06), que compreende os seguintes sítios anatômicos: mucosa labial e jugal, soalho de boca, rebordo alveolar e gengiva, dois terços anteriores da língua (anteriores às papilas circunvaladas), palato duro e trígono retromolar. Foram excluídos casos de CE da região de orofaringe, que inclui palato mole, tonsilas palatinas, pregas do palatoglosso, valécula e parede posterior da faringe, exceto casos localizados no terço posterior da língua (base de língua) e palato mole, que foram incluídos porque se localizam em áreas que podem ser acessadas para a realização de biópsia intraoral pelo cirurgião dentista sob anestesia local. Também foram excluídos casos notificados como outras neoplasias malignas da cavidade oral ou casos de CE sem o diagnóstico comprovado por meio de análise histopatológica.

Para a análise dos dados, foi adotado um método de comparação de dados relativos a incidência do CE com uma série de casos diagnosticados em um período de seis anos (análise quantitativa). Para isso, foram comparados os dados do 
Registro de Câncer de Base Populacional (RCBP) referentes aos anos 2008-2013 com os casos diagnosticados em um serviço público municipal de Palmas (Centro de Especialidades Odontológicas) dos anos 2014-2019. O Instituto Nacional de Câncer (INCA) foi contatado para colhimento de informações a respeito do RCBP e entendimento do funcionamento do Registro Hospitalar de Câncer (RHC), pois o RHC também serve como base de dados para compor as estatísticas do RCBP. Os dados foram comparados por análise descritiva conforme tabelas 1 e 2 .

\section{Tabela 1}

Dados do Registro de Câncer de Base Populacional sobre o carcinoma epidermoide oral (carcinoma escamocelular), no período compreendido entre 2013-2018.

\begin{tabular}{l|l|l|l|l|l}
\hline \multicolumn{1}{c|}{ Caso } & \multicolumn{1}{c|}{ Sexo } & \multicolumn{1}{c|}{ Idade } & \multicolumn{1}{c|}{ Raça } & \multicolumn{1}{c|}{ CID } & \multicolumn{1}{c}{ Localização } \\
\hline 1 & Masculino & 50 & Parda & C00 & Lábio inferior \\
\hline 2 & Masculino & 55 & Parda & C01 & Base da língua \\
\hline 3 & Feminino & 55 & Parda & C01 & Base da língua \\
\hline 4 & Feminino & 51 & Parda & C01 & Base da língua \\
\hline 5 & Masculino & 47 & Branco & C01 & Base da língua \\
\hline 6 & Masculino & 49 & Parda & C01 & Base de língua \\
\hline 7 & Masculino & 48 & - & $\mathrm{C} 02$ & Bordo da lingua \\
\hline 8 & Masculino & 65 & - & $\mathrm{C} 02$ & $2 / 3$ anteriores da língua \\
\hline 10 & Masculino & 54 & - & $\mathrm{C} 02$ & Língua \\
\hline 11 & Feminino & 58 & Parda & $\mathrm{C} 02$ & Língua \\
\hline 12 & Feminino & 83 & Parda & $\mathrm{C} 05$ & Palato mole \\
\hline 13 & Masculino & 72 & Parda & $\mathrm{C} 05$ & Palato mole \\
\hline 14 & Masculino & 47 & Parda & $\mathrm{C} 06$ & Área retromolar \\
\hline 15 & Masculino & 56 & Parda & C06 & Área retromolar \\
\hline 16 & Feminino & 48 & Parda & C06 & Área retromolar \\
\hline
\end{tabular}

Legenda: C00: neoplasia maligna do lábio; C01: neoplasia maligna de base de língua; C02: neoplasia maligna de outras partes e de partes não especificadas da língua; C03: neoplasia maligna de gengiva; C05: neoplasia maligna do palato; C06: neoplasia maligna de outras partes e de partes não especificadas da boca

\section{Tabela 2}

Dados do Centro de Especialidades Odontológicas (CEO) sobre o carcinoma epidermoide oral (carcinoma escamocelular), no período compreendido entre 2014-2019.

\begin{tabular}{l|l|l|l|l|l} 
Caso & Sexo & Idade & Raça / Cor & CID & Localização \\
\hline 1 & Masculino & 54 & Branca & C00 & Lábio inferior \\
\hline 2 & Feminino & 50 & Branca & C00 & Lábio inferior \\
\hline 3 & Masculino & 77 & Branca & C00 & Lábio inferior \\
\hline 4 & Masculino & 36 & - & C02 & Borda lateral de língua \\
\hline 5 & Masculino & 53 & Pardo & C02 & Borda de língua \\
\hline 6 & Feminino & 34 & Branca & C02 & Ventre de língua \\
\hline 7 & Masculino & 51 & - & C03 & Mucosa jugal e orofaringe \\
\hline 8 & Feminino & 74 & Branca & C05 & Palato duro \\
\hline 9 & Masculino & 53 & Branca & C06 & Rebordo alveolar \\
\hline 10 & Feminino & 52 & Negra & C06 & Rebordo alveolar \\
\hline 11 & Masculino & 71 & Branco & C06 & Assoalho bucal \\
\hline 12 & Masculino & 54 & Indígena & C06 & Assoalho bucal \\
\hline 13 & Masculino & - & Pardo & C06 & Rebordo alveolar \\
\hline 14 & Feminino & 41 & Branca & C06 & Região de trígono retromolar \\
\hline 15 & Masculino & 56 & Branco & C06 & Mucosa jugal \\
\hline 16 & Masculino & 51 & Pardo & C06 & Mucosa jugal \\
\hline
\end{tabular}

Legenda: C00: neoplasia maligna do lábio; C01: neoplasia maligna de base de língua; C02: neoplasia maligna de outras partes e de partes não especificadas da língua; C03: neoplasia maligna de gengiva; C05: neoplasia maligna do palato; C06: neoplasia maligna de outras partes e de partes não especificadas da boca

Os dados obtidos foram editados em Microsoft ${ }^{\circledast}$ Excel $^{\circledR}$ e tabulados no programa Stata ${ }^{\circledR}$, versão 11.0. Efetuou-se a análise descritiva dos dados, em relação as variáveis sexo, raça e CID (localização da lesão), sendo feita a comparação dos casos diagnosticados no RCBP (2013-2018) e no CEO Palmas (20142019).

\section{RESULTADOS}

Os resultados do presente trabalho podem ser verificados nas Tabelas 1 e 2 e nos Gráficos 1, 2 e 3.

Foram encontrados 16 casos notificados em um período de 6 anos no RCBP e foram encontrados também 16 casos em um período de 6 anos em um único serviço público de Palmas, conforme Tabelas 1 e 2.

Em relação a variável sexo, observou-se também uma igualdade no número de casos notificados no RCBP (2008-2013) e no CEO (2014-2019), com cinco casos de CE verificados em pacientes do sexo feminino e 11 casos em pacientes do sexo masculino, em ambos (Gráfico 1).

\section{Gráfico 1}

Distribuição dos casos de carcinoma epidermóide notificados no RCBP e no CEO em função da variável Sexo

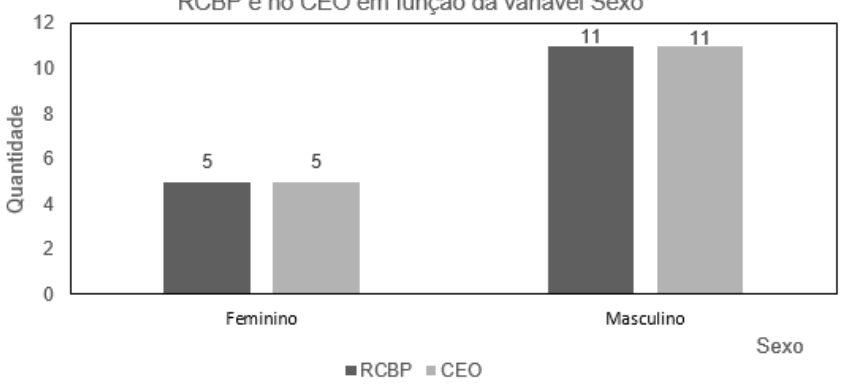

Em relação a variável raça, houve diferença significativa entre as raças parda e branca em cada local, sendo que no RCBP (2008-2013) houve um predomínio de pacientes de raça parda, enquanto no CEO houve predomínio de pacientes de raça branca; já as outras raças (negra e indígena) tiveram quantidade semelhante de casos (Gráfico 2).

\section{Gráfico 2}

Distribuição dos casos de carcinoma epidermóide notificados no RCBP e no CEO em função da variável Raça

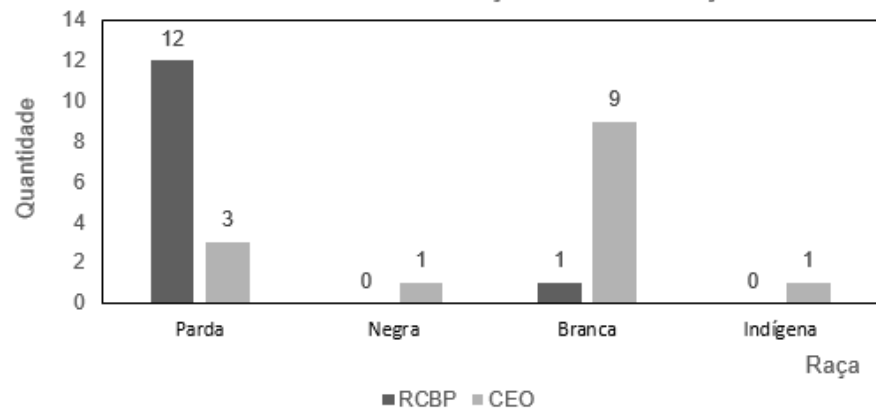

Em relação a variável CID (localização / tipo da lesão), observou-se que no CEO (2014-2019) houve mais casos notificados com CID C00 (neoplasia maligna de lábio) e C06 (neoplasia maligna de outras partes e de partes não especificadas da boca), enquanto no RCBP (2008-2013) houve predomínio de casos notificados em base de língua (CID C01) e outras partes e partes não especificadas da língua (CID CO2) (Gráfico 3).

\section{Gráfico 3}


Distribuição dos casos de carcinoma epidermóide notificados no RCBP e no CEO em função da variável CID

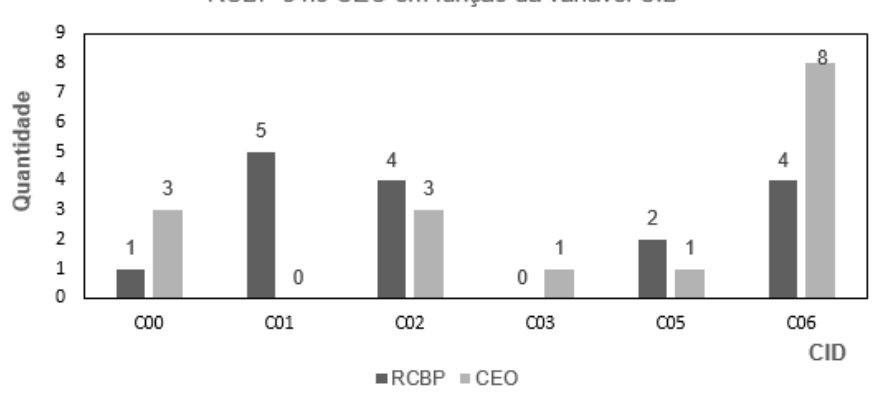

\section{DISCUSSÃo}

Os dados dessa pesquisa permitem analisar genericamente as características dos pacientes acometidos pelo carcinoma epidermoide oral (CE) em Palmas, Tocantins, bem como comparar variáveis como sexo, raça e localização das lesões neoplásicas, em um intervalo de tempo de seis anos. Os dados de Registro de Câncer de Base Populacional (RCBP) que constam no presente trabalho (2008-2013) compreendem o último registro divulgado pelo INCA para a cidade de Palmas, Tocantins, e envolvem todos os casos notificados nos serviços de referência do município no período mencionado. Os dados do Centro de Especialidades Odontológicas (CEO 2014-2019) ainda não constam no RCBP e envolvem todos os casos de CE diagnosticados em um único serviço de referência do município, em um intervalo de tempo posterior, porém equivalente ao verificado no RCBP. Embora a quantidade de pacientes com CE diagnosticados no CEO não reflita a totalidade de pacientes acometidos pela doença no município de Palmas, a comparação com os dados do RCBP permitiu analisar as variáveis mencionadas para um melhor entendimento do perfil de pacientes que convivem com o CE. Em relação à variável sexo, não houve nenhuma diferença entre os sexos quando se compara os dados do RCBP e CEO, uma vez que a quantidade de pacientes do sexo feminino e masculino acometidos no intervalo de tempo analisado em ambos foi a mesma (Gráfico 1). Nos dois casos houve prevalência entre homens acima de 40 anos (Tabelas 1 e 2), o que corrobora com os dados epidemiológicos da OMS e do INCA quando se considera o grupo de risco para o desenvolvimento do $\mathrm{CE}{ }^{4}$. Embora alguns autores tenham relatado uma mudança em relação à idade dos pacientes acometidos (cada vez mais jovens) bem como um aumento de incidência de CE em mulheres, na cidade de Palmas, Tocantins, pode-se verificar que ainda há maior predominância de CE em pacientes do sexo masculino acima de 40 anos, conforme classicamente descrito na literatura ${ }^{4}$.

Em relação à raça, no RCBP houve uma predominância de casos de pacientes de raça parda, enquanto no CEO houve uma predominância de pacientes da raça branca, sendo a raça um fator autodeclarado pelo indivíduo (Gráfico 2). É importante destacar que no CEO houve mais casos de carcinoma de lábio (CID CO0) quando comparados ao RCBP (Gráfico 3), e o carcinoma de lábio ocorre principalmente em pessoas de raça branca, pois seu aparecimento está diretamente relacionado à exposição solar crônica naqueles que possuem menos proteção natural contra os efeitos nocivos da radiação ultravioleta (indivíduos de pele clara).
Quanto à localização / tipo da lesão (CID), houve grande diferença no perfil de pacientes acometidos. No CEO não foi identificado nenhum paciente com CE em base de língua (CID C01), enquanto no RCBP foram notificados cinco casos (Gráfico 3). Quanto ao CE de outras partes e de partes não especificadas da boca (CID C06), no CEO Palmas foram identificados o dobro de casos quando comparados ao RCBP (Gráfico 3). Essa diferença pode ter ocorrido pelo fato de que o CEO é um serviço apenas de diagnóstico, em que os pacientes suspeitos são submetidos a biópsias incisionais, em ambiente exclusivamente ambulatorial, sob anestesia local, enquanto no outro serviço de referência (HGP), que consta nos dados de notificação do RCBP, realiza-se, também, o tratamento dos pacientes por meio de biópsias excisionais, em ambiente hospitalar, sob anestesia geral, o que permite um melhor acesso às lesões localizadas em orofaringe. Além disso, os pacientes encaminhados ao serviço do HGP contam com o atendimento de outros profissionais de saúde, como médicos de cabeça e pescoço. Essas diferenças entre o perfil de atendimento aos pacientes e o perfil de profissionais envolvidos nos atendimentos talvez possa influenciar nas variações de localização das lesões diagnosticadas no CEO quando comparadas à localização das lesões notificadas no RCBP.

Por fim, a análise comparativa da quantidade de casos notificados por meio do RCBP (2008-2013) e em um único serviço de referência para atendimento aos pacientes com CE em Palmas (CEO 2014-2019), em um mesmo intervalo de tempo, nos leva a inferir duas hipóteses: (1) embora o RCBP seja um meio confiável de registro de câncer, os dados referentes à incidência de CE na população de Palmas podem estar subestimados, já que, em um mesmo intervalo de tempo, um único serviço da cidade (CEO) registrou o mesmo número de casos notificados no último RCBP; ou (2) houve um aumento real incidência de CE na população de Palmas, o que poderá ser evidenciado com a próxima divulgação de dados de registro do RCBP no período correspondente aos registros do CEO (20142019). Novos estudos se fazem necessários para confirmar ou excluir as hipóteses mencionadas.

\section{CONCLUSÃo}

Diante do exposto, ressalta-se a importância dos exames anatomopatológicos para a confirmação diagnóstica e a necessidade da notificação do CE pelos profissionais aos setores responsáveis, o que permite uma ampla coleta de dados epidemiológicos pelo Ministério da Saúde e consequentemente possibilita uma melhoria das políticas públicas para o enfrentamento da doença, com a implementação e a manutenção dos serviços de referência para diagnóstico e o tratamento.

\section{REFERÊNCIAS BIBLIOGRÁFICAS}

1. El- Naggar AK, Chan JKC, Grandis JR, Takata T, Slootweg PJ. WHO Classification of Head and Neck Tumours. 4th ed. International Agency for Research on Cancer (IARC); 2017 
2. Chi AC, Day TA, Neville BW. Oral cavity and oropharyngeal squamous cell carcinoma - an update. CA Cancer J Clin. 2015;65(5):401-21

3. Deschler DG, Richmon JD, Khariwala SS, Ferris RL, Wang MB. The "new" head and neck cancer patient-young, nonsmoker, nondrinker, and HPV positive: evaluation. Otolaryngol Head Neck Surg. 2014;151: 375-380

4. Ferlay, J. Ervik $M$, Lam F, Colombet $M$, Mery L, Piñeros $M$, Znaor A, Soerjomataram I, Bray F. Cancer today. Lyon, France: International Agency for Research on Cancer, 2018. (IARC CAncerBase, n. 15). Available at: https://publications.iarc.fr/Databases/ larcCancerbases/Cancer-Today-Powered-By-GLOBOCAN-2018-2018. Access in: 9 Sep. 2019.

5. Instituto Nacional do Cancer (INCA), Ministério da Saúde: Estimativa 2020: Incidência de Câncer no Brasil. Brasil. Brasil, INCA, 2019. https://www.inca.gov.br/sites/ufu.sti.inca.local/files/media /document/estimativa-2020-incidencia-de-cancer-nobrasil.pdf/. Acesso em 22 de novembro 2020.

6. American Cancer Society. Cancer facts \& figures 2019. Atlanta: American Cancer Society, 2019

7. Registros de Câncer de Base Populacional". INCA - Instituto Nacional de Câncer, https://www.inca.gov.br/numeros-decancer/registro-de-cancer-de-base-populacional. Acesso: 22 de novembro de 2020 